\title{
Calidad de Vida Laboral, Catastrofismo y Aceptación del Dolor Crónico Osteomuscular en Mujeres Trabajadoras
}

\section{QUALITY OF WORKING LIFE, CATASTROPHIZING AND ACCEPTANCE OF CHRONIC MUSCULOSKELETAL PAIN IN WORKING WOMEN}

\section{Cecilia Andrea Ordóñez-Hernández', Mónica Isabel Contreras-Estrada², Raquel González-Baltazar ${ }^{3}$}

1. Maestra en Salud Ocupacional. Doctora en Ciencias de la Salud en el Trabajo, Universidad de Guadalajara.

2. Profesora Investigadora de la Maestría en Salud Ocupacional y del Doctorado en Ciencias de la Salud en el Trabajo, Universidad de Guadalajara.

3. Docente Titular "C" Doctorado en Ciencias de la Salud en el Trabajo Universidad de Guadalajara.

\section{RESUMEN}

Objetivo: Determinar la asociación de la calidad de vida laboral con el catastrofismo y la aceptación del dolor crónico osteomuscular en mujeres trabajadoras. Material y métodos: Estudio transversal, analítico, correlacional con 88 trabajadoras que consultaron por dolor crónico en un hospital de Guadalajara. Los instrumentos fueron CVTGOHISALO, Escala de catastrofización del dolor y Cuestionario de aceptación del dolor. Para la prueba de hipótesis se usó $\mathrm{r}$ de Pearson. Resultados: Las trabajadoras reportaron niveles bajos en todas las dimensiones de calidad de vida laboral, alta catastrofización y baja aceptación global del dolor. Se encontró relación estadística inversa entre las dimensiones soporte institucional, Integración al puesto de trabajo y Bienestar logrado a través del trabajo con la alta catastrofización del dolor (p 0,025, 0,003, 0,007) y relación directa entre las dimensiones soporte institucional, integración al puesto de trabajo, satisfacción por el trabajo y desarrollo personal del trabajador con la alta aceptación del dolor (p 0,008, 0,007, 0,048, 0,001). Conclusión: La baja satisfacción con la calidad de vida laboral favorece la catastrofización del dolor crónico osteomuscular y la alta satisfacción con la calidad de vida laboral favorece la aceptación del dolor crónico osteomuscular.

(Ordóñez C, Contreras M, González R, 2017. Calidad de Vida Laboral, Catastrofismo y Aceptación del Dolor Crónico Osteomuscular en Mujeres Trabajadoras. Cienc Trab. Ene-Abr; 19 [58]: 26-30).

Palabras clave: CALIDAD DE VIDA LABORAL, DOLOR, MUJER.

\section{ABSTRACT}

Aim: To determine the association of the quality of working life with catastrophizing and acceptance of chronic pain in musculoskeletal working women. Material and Methods: Cross-sectional, analytical, correlational 88 workers who consulted for chronic pain in a hospital in Guadalajara Study. The instruments were CVT GOHISALO, Pain catastrophizing scale and CPAQ Pain acceptance questionnaire. For hypothesis testing is used Pearson's r. Results: The workers reported low levels in all dimensions of quality of working life, high catastrophizing and low overall acceptance of pain. Statistical inverse relationship between the dimensions institutional support, integration into the workplace and Welfare achieved through work with high catastrophizing pain ( 0 0,025, 0,003, 0,007) and direct relationship between the dimensions institutional support, integration post was found work, job satisfaction and personal development worker with the high acceptance of pain (p 0,008, 0,007, 0,048, 0,001). Conclusion: The low satisfaction with the quality of work life favors of chronic musculoskeletal pain catastrophizing and high satisfaction with the quality of work life favors acceptance of chronic musculoskeletal pain.

Key words: QUALITY OF WORK LIFE, PAIN, WOMEN.

\section{INTRODUCCIÓN}

Hoy en día la salud en el trabajo se ha convertido en una necesidad y en un soporte fundamental de los sistemas de seguridad social de los países, por su impacto sobre la calidad de vida tanto

Correspondencia / Correspondence:

Cecilia Andrea Ordóñez Hernández

Jardines de los amigos 576. Fraccionamiento Jardines de Verano

Código postal 44970

Guadalajara, Jalisco. México

Tel: (01) 443336126278

e-mail: andrea-ordonez@hotmail.com

Recibido: 10 de Abril de 2016 / Aceptado: 31 de Enero de 2017 del trabajador como de su familia; en este sentido, las características del trabajo deberían además ofrecer satisfacción, bienestar y desarrollo personal, determinantes de la calidad de vida laboral. El concepto de calidad de vida laboral es multidimensional, pretende combinar el trabajo con el individuo, lograr que las experiencias individuales sean satisfactorias y además apunten a los objetivos de la organización; en otras palabras, la interacción del individuo con el entorno en el que se desenvuelve ${ }^{1}$, lo cual aplica también a las circunstancias en las cuales el trabajador no goce de buena salud.

Las cifras del Instituto Mexicano del Seguro Social indican que la prevalencia de dolor crónico en la población general de mujeres es de 39,6\% y se presenta desde los 15 hasta los 86 años. Las principales causas son artritis, fibromialgia, cefaleas, cervicalgias y dorsalgias, encontrando un incremento en los grupos de mayor edad tanto en la prevalencia como en la intensidad, alcanzando el 41,5\%. La consulta de adultos entre 20 y 59 años repre- 
sentó el 52\% de la atención médica familiar y, de estos, 13\% consultó por una enfermedad asociada potencialmente a dolor crónico. Por otro lado, un estudio multicéntrico realizado en España ${ }^{8}$ en 2013, que pretendió conocer el perfil de los pacientes con dolor crónico músculo-esquelético, señala que de los 1325 pacientes que consultaron, $62,9 \%$ son mujeres, con una media de edad de 59,8 años.

Por sus características, el dolor osteomuscular afecta las articulaciones y su musculatura adyacente, en algunos casos suele ser crónico y difícil de manejar y extenderse por periodos prolongados de tiempo. ${ }^{2-4}$ La consecuencia es la limitación del movimiento y la dificultad para la realización de actividades cotidianas y laborales, dando pie a la aparición de afecciones en la salud psicológica, como depresión y ansiedad, que empeoran aún más el dolor. ${ }^{5-7,9}$

Dada la importancia de la actividad laboral en la vida de todo trabajador y el tiempo que se le dedica por día, el interés giró en torno a la posible influencia de la calidad de vida laboral sobre la respuesta de una trabajadora con dolor crónico osteomuscular a su situación de enfermedad; de esta manera nos planteamos como objetivo determinar la relación entre la satisfacción con la calidad de vida laboral y la catastrofización del dolor crónico en mujeres trabajadoras.

\section{MATERIAL Y MÉTODOS}

Se utilizó un diseño cuantitativo de carácter analítico, transversal y correlacional. La población estuvo conformada por trabajadoras federales mexicanas con dolor crónico osteomuscular, con consultas subsecuentes por la misma causa y diagnósticos confirmados, que asistieron a un consultorio de los servicios de ortopedia y neurocirugía de un hospital de tercer nivel de la ciudad de Guadalajara en julio de 2014. Se obtuvo una muestra de 88 trabajadoras seleccionadas a través de un muestreo no probabilístico por cuotas, considerando los siguientes criterios de selección: ser mujer, activa laboralmente, servidora pública, presentar dolor crónico osteomuscular mayor a 6 meses, no estar calificada en estado de invalidez y querer participar voluntariamente. Considerando que el tipo de trabajadoras que asistió a la consulta externa fue azaroso, no se consideraron otras variables como la edad, el grado de cronicidad del dolor y el tipo de trabajo dada la alta variabilidad en las características de las trabajadoras que se incluyeron en el estudio.

Para la recolección de la información se utilizó el Instrumento de medición de la calidad de vida CVT-GOHISALO ${ }^{10}$, el cual cuenta con validez de contenido, constructo y criterio, con índice Alpha de Cronbach de 0,9527. Está constituido por 74 ítems, que se integran en 7 dimensiones:

- Soporte institucional para el trabajo, definido por los elementos del puesto de trabajo que son aportados por la institución como la supervisión, el apoyo por parte de los superiores, la forma de evaluación y el grado de autonomía.

- Seguridad en el trabajo, entendida como las condiciones del empleo que ofrecen al trabajador firmeza en su relación con la institución e incluye los procedimientos de trabajo, el salario, los derechos contractuales y la capacitación para el trabajo.

- Integración al puesto de trabajo, se define como la inserción del trabajador al trabajo como una más de sus partes e incluye el sentido de pertenencia, la motivación y el ambiente laboral.
- Satisfacción por el trabajo, entendida como la sensación global de agrado que el trabajador tenga por su empleo incluye la dedicación al trabajo, el orgullo por la institución, la participación en el trabajo, el reconocimiento y la autovaloración.

- Bienestar logrado a través del trabajo: se entiende como la satisfacción con el disfrute de bienes adquiridos a través del trabajo, relacionados con la vivienda, la salud, la nutrición.

- Desarrollo personal del trabajador: se define como la percepción de mejora de aspectos personales relacionados con la actividad laboral como logros, expectativas y seguridad personal.

- Administración del tiempo libre determinado por la forma en que se disfruta del horario en que no se trabaja, considera la planificación del tiempo libre y el equilibrio entre el trabajo y la vida familiar.

Para la calificación e interpretación de los resultados se utilizó el baremo que determina niveles bajos, medios y altos en cada una de las dimensiones a partir del percentil 50 con desviación estándar de 10. Se evaluó nivel alto por encima del percentil $60 \mathrm{y}$ nivel bajo por debajo del percentil 40. Se consideró, por tanto, satisfecho con cada una de las dimensiones los valores por encima del percentil 60 e insatisfecho los valores inferiores, agrupando los niveles medios y los bajos de satisfacción.

La Escala de Catastrofización (PCS) ${ }^{11}$ versión española, cuestionario autoadministrable de 13 items que evalúa tres dimensiones: Rumiación ("no puedo dejar de pensar en lo mucho que duele"), Magnificación ("es horrible y siento que me abruma”) y Desesperanza ("es horrible y siento que es más fuerte que yo"); cada item puntúa desde 0 (nada) hasta 4 (siempre) obteniendo una puntuación máxima de 52; el intervalo teórico del instrumento se sitúa entre 13 y 62, puntajes bajos indican escasa catastrofización y puntajes altos elevada catastrofización. Su Coeficiente de Cronbach es 0,79 y su Coeficiente de Correlación intra clase 0,84.

El Cuestionario de aceptación del dolor (CPAQ), Validación de la versión española por Rodero, García-Campayo, Casanueva, López, Serrano y Luciano (2010). Este instrumento es un cuestionario autoadministrable compuesto de 20 ítems que se valoran en una escala de Likert desde 0 (nunca es verdad) hasta 6 (siempre es así), obteniendo tres puntuaciones: una total y dos subescalas, una en la dimensión implicación en las actividades, la cual se refiere al grado de afectación que el dolor produce en la vida de quien lo padece y la otra dimensión apertura al dolor, relacionado con el grado de aceptación del dolor que se ha intentado modificar sin éxito (González, Fernández, Torres 2010).

El análisis de validación del CPAQ español mostró que tienen una buena fiabilidad "test-retest" (con un coeficiente de correlación intra clase de 0,83) y fiabilidad de la consistencia interna (Cronbach de 0,83). Las puntuaciones del CPAQ español se correlacionaron significativamente con la intensidad del dolor, la ansiedad, la depresión, el catastrofismo del dolor, el estado de salud y la discapacidad física y psicosocial.

Se abordó individualmente a cada trabajadora, en la sala de espera antes de la consulta médica, se presentó el proyecto, objetivos, metodología, forma de participación y se leyó el consentimiento informado aclarando las dudas que surgieron. Se procedió a la aplicación de los instrumentos a las trabajadoras seleccionadas, en un tiempo estimado de 20 min.

Los datos fueron registrados en una base de datos en Excel (Microsoft) y luego exportados al programa estadístico Statistical 
Package for the Social Sciences (SPSS) versión 21. Para la comparación de las dimensiones de la calidad de vida laboral con la catastrofización se utilizó el estadístico $r$ de Pearson.

El estudio tuvo la autorización del comité de ética médica de la institución donde se desarrolló la investigación y consideró para su diseño la Ley General de Salud de México (1986), Título Segundo "De los aspectos éticos de la investigación en seres humanos", que resalta el criterio de respeto a la dignidad de los participantes y la protección de sus derechos y bienestar y exige la protección de la privacidad. Según esta norma, este estudio es considerado como una investigación sin riesgo, se utilizaron cuestionarios y entrevistas diseñadas con anticipación y no se intervino o modificó intencionadamente ninguna variable fisiológica o psicológica de los participantes.

Para el control de sesgos, se explicó detalladamente a las participantes cada uno de los instrumentos y se resaltó la importancia de responder honestamente las preguntas. Con el fin de garantizar la confidencialidad y privacidad de los datos, las trabajadoras se identificaron alfa numéricamente según número de cuestionario que correspondió al orden de diligenciamiento.

\section{RESULTADOS}

Se encontró que la edad promedio es 50,9 +/- 8,8 años, 39,8\% realizó estudios superiores, 50\% reportó estar casada, 64,7\% manifestó tener entre 1 y 3 hijos, 86,4\% indicó doble presencia, es decir, realiza las labores domésticas además de trabajar. En cuanto al cargo desempeñado, 34,1 expresó ser docente y 26,1 enfermera. El $52,3 \%$ de las mujeres participantes comparte la responsabilidad económica de sus hogares.

Los dolores más prevalentes fueron: artrosis de rodilla (17\%), hernia lumbar $(12,5 \%)$ y hernia cervical y lumbar concomitante $(12,5 \%)$, los cuales registraron una cronicidad del dolor, en un rango de 6 meses a 31 años con promedio de 5,8 +/- 5,6 años de duración del dolor.

La satisfacción con la calidad de vida laboral se midió en siete dimensiones, las cuales presentaron niveles bajos en todos los casos, como se puede observar en la Tabla 1, destacándose la insatisfacción en el Bienestar logrado a través del trabajo $(98,9 \%)$, determinado por escasos beneficios percibidos en nutrición, salud y vivienda otorgados por el trabajo. Asimismo, se encontró baja satisfacción con el soporte institucional (75\%), es decir, se percibe falta de apoyo por parte de los superiores, mala supervisión laboral y pocas oportunidades de promoción; 85,2\% de las trabajadoras manifestó baja satisfacción con la dimensión seguridad en el trabajo, la cual se relaciona con pocos derechos contractuales, poca capacitación en el trabajo e inconformidad con el diseño de sus procedimientos de trabajo.

La baja satisfacción por el trabajo encontrada en $86,4 \%$ de explica por el poco reconocimiento y autovaloración percibido, 87\% de las trabajadoras manifestaron baja satisfacción con la dimensión Integración en el puesto de trabajo explicado por falta de motivación, de sentido de pertenencia y mal ambiente laboral; 90,9\% manifiestan baja satisfacción con la administración del tiempo libre, determinado por la poca posibilidad de planificar el tiempo libre y la dificultad para establecer el equilibrio entre la relación familia trabajo. Se encontró baja satisfacción con la dimensión Desarrollo personal del trabajador en 79,5\% de las mujeres, relacionada con las escasas expectativas de mejora en la seguridad personal a través del trabajo.
Tabla 1.

Dimensiones de la Calidad de Vida Laboral.

\begin{tabular}{|c|c|c|c|c|}
\hline \multirow{2}{*}{$\begin{array}{l}\text { Variable } \\
\text { Dimensiones de calidad de vida labora }\end{array}$} & \multicolumn{2}{|c|}{ Alta satisfacción } & \multicolumn{2}{|c|}{ Baja satisfacción } \\
\hline & & & & \\
\hline Soporte Institucional & 22 & 25,0 & 66 & 75,0 \\
\hline Seguridad en el trabajo & 13 & 14,8 & 75 & 85,2 \\
\hline Integración al puesto de trabajo & 11 & 12,5 & 77 & 87,5 \\
\hline Satisfacción por el trabajo & 12 & 13,6 & 76 & 86,4 \\
\hline Bienestar logrado a través del trabajo & 1 & 1,1 & 87 & 98,9 \\
\hline Desarrollo personal del trabajador & 18 & 20,5 & 70 & 79,5 \\
\hline Administración del tiempo libre & 8 & 9,1 & 80 & 90,9 \\
\hline
\end{tabular}

Tabla 2.

Dimensiones del catastrofismo del dolor crónico OTM de mujeres trabajadoras.

\begin{tabular}{lcccc} 
Variable & \multicolumn{2}{c}{ Alto } & \multicolumn{2}{c}{ Bajo } \\
\hline Catastrofización del dolor & $\mathrm{n}$ & $\%$ & $\mathrm{n}$ & $\%$ \\
Rumiación & 82 & 93,2 & 6 & 6,8 \\
Magnificación & 78 & 88,6 & 10 & 11,4 \\
Desesperanza & 81 & 92 & 7 & 8 \\
Aceptación del dolor & 75 & 85,2 & 13 & 14,7 \\
Apertura al dolor & 32 & 36,4 & 56 & 63,6 \\
Implicación en las actividades & 50 & 56,8 & 38 & 43,2 \\
& 40 & 45,5 & 48 & 54,5
\end{tabular}

Se encontraron niveles altos en las tres dimensiones evaluadas de la catastrofización del dolor y en su calificación global $(93,2 \%)$. Puede verse con mayor detalle que $88,6 \%$ tiene alta rumiación, entendiéndose este término como un mecanismo cognitivo de afrontamiento, en el cual las mujeres focalizan su atención en aspectos difíciles y negativos con respecto al dolor; 92\% refiere alta magnificación, traducido en pensamientos pesimistas y agravadores del hecho de padecer el dolor y la desesperanza (94,3\%), es decir, sentirse rendida frente a la presencia de dolor. Este comportamiento en general ha demostrado que puede entorpecer los tratamientos y potencialmente extender los periodos de incapacidad laboral. (Tabla II).

Se utilizó el estadístico $r$ de Pearson para correlacionar cada una de las dimensiones de la calidad de vida laboral, con el valor global de la catastrofización y con cada una de sus dimensiones. Se encontró relación inversa de las dimensiones soporte Institucional con la magnificación, la desesperanza y la calificación global de la catastrofización ( $\mathrm{p}$ 0,045, 0,013, 0,025); de la dimensión Integración al puesto de trabajo con la rumiación, magnificación, desesperanza y con la calificación global catastrofización (p 0,007, 0,006, 0,005 y 0,003); de la dimensión bienestar logrado a través de trabajo con la rumiación, magnificación, desesperanza y catastrofización global (p 0,008, 0,027, 0,009, 0,007), respectivamente, y de la dimensión Administración del tiempo libre con el desesperanza (p 0,032) (Tabla III). Cabe resaltar que los valores de r encontrados no son muy cercanos ni a $1 \mathrm{ni}$ a -1, indicando que, aunque la correlación existe, el lazo de asociación es débil.

\section{DISCUSIÓN}

Los resultados de esta investigación indican asociación negativa entre las variables satisfacción con la calidad de vida laboral y catastrofización del dolor crónico osteomuscular; cuando la satisfacción con la calidad de vida laboral disminuye, la catastrofización del dolor aumenta. Una vez el dolor se presenta, afecta notoriamente el 
Tabla 3.

Prueba de la comprobación de hipótesis con el estadístico $r$ de Pearson, entre las dimensiones de la CVL y la catastrofización del dolor crónico OTM de las trabajadoras en estudio.

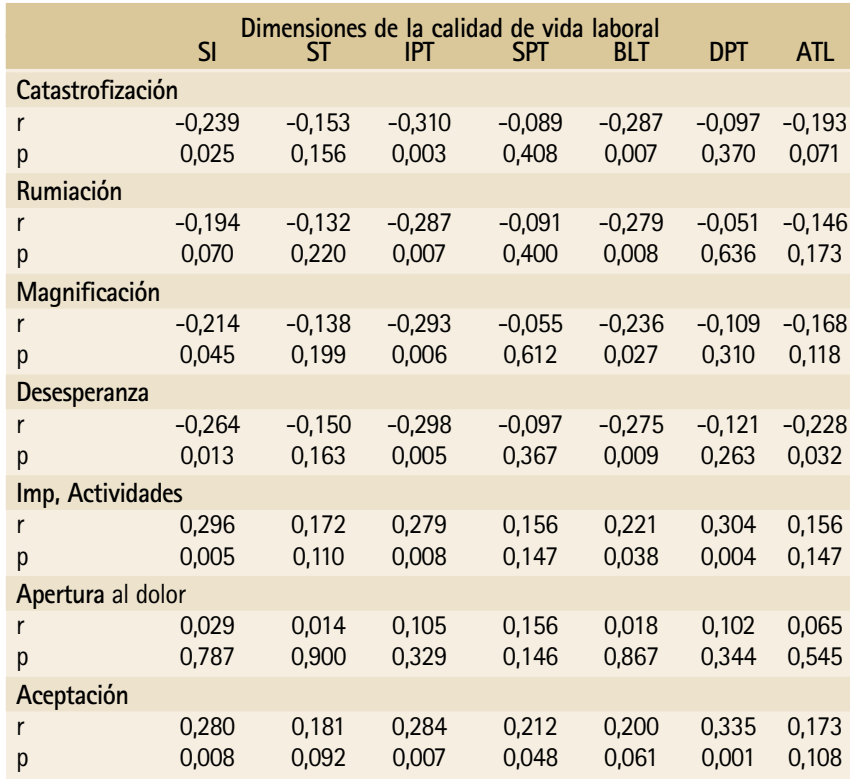

Fuente: Cuestionario CVT GOHISALO, CPAQ y PCS aplicado a las trabajadoras con dolor crónico OTM. SI: Soporte Institucional, ST: Seguridad en el trabajo, IPT: Integración al puesto de trabajo, ST: Satisfacción por el trabajo, BLT: Bienestar logrado a través del trabajo, DPT: Desarrollo personal del trabajador, ATL: Administración del tiempo libre.

desarrollo cotidiano de las tareas tanto domésticas como laborales, como lo evidenció un estudio realizado en Colombia ${ }^{12}$ en el que trabajadores con dolor osteomuscular manifestaron que el dolor interfiere con sus actividades de la vida diaria e impacta negativamente la realización del trabajo normal $(82,7 \%)$, estado de ánimo (62\%), afectación del sueño (48,2\%); igualmente en Brasil $2012^{13}$, se encontró asociación positiva entre la intensidad del dolor músculo-esquelético y la reducción de la capacidad para el trabajo en 498 trabajadores de enfermería.

La primera causa de dolor crónico osteomuscular para las trabajadoras participantes es la artrosis de rodilla (17\%), seguida por hernia cervical y lumbar concomitante $(12,5 \%)$ tal como lo evidencia un estudio realizado en Venezuela $2009^{14}$, que describe las patologías más frecuentes asociadas al sexo femenino y reporta la osteoartritis y la artritis reumatoide como primeras causas de dolor. También señalan que las mujeres tienen mayor probabilidad que los hombres de presentar varios tipos de dolor en forma simultánea, lo cual conduce a mayor discapacidad y disfunción psicoló- gica, como es el caso de las trabajadoras participantes que muestran alta catastrofización del dolor $(93,2 \%)$.

La relación inversa entre las dimensiones soporte institucional e Integración al puesto de trabajo con la catastrofización del dolor (p 0,025 y 0,003), es congruente con un estudio realizado en Venezuela en $2009^{15}$, que encontró relación de los síntomas músculo-esqueléticos de cuello $(82,68 \%)$ y espalda $(24,4 \%)$, con estrés laboral derivado de problemas con la supervisión y malas relaciones con los compañeros de trabajo.

Asimismo, un estudio realizado en México ${ }^{16}$ sobre acoso laboral y fibromialgia señala que las 29 mujeres participantes reportaron sentimientos de humillación y desprecio provocados por jefes que ellas calificaron como hiperquinéticos, autoritarios, con sindrome de bipolaridad, soberbios, obsesivos, lo que exacerbó los síntomas de la fibromialgia, mostrando relación con la experiencia de maltrato en el medio laboral.

Según García-Campayo y Rodero ${ }^{17}$, “[...] la aceptación del dolor implica tener contacto con experiencias desagradables o dolorosas sin que esto influya en la conducta [...] incluso cuando las sensaciones sean intensas, no es necesario ignorarlas, eliminarlas o controlarlas para llevar una vida plena”. La aceptación del dolor permite a las personas mejorar el pronóstico de su dolor y percibir una mejor calidad de vida tanto en el trabajo como fuera de él. En este estudio, 63,6\% de las trabajadoras refirieron un nivel global bajo de aceptación del dolor; sin embargo, al analizar las dimensiones que conforman este constructo, se observa que la apertura al dolor es alta en 56,8\% y la implicación en las actividades es baja en 54,5\%. Esto puede explicarse por el hecho de que las mujeres continúan trabajando y participando en actividades tanto familiares como laborales a pesar de referir dolor. En este caso las responsabilidades y el apoyo familiar son la principal motivación para que estas trabajadoras continúen laborando ${ }^{18}$, no así el medio laboral, el cual se presenta hostil y excluyente. ${ }^{19}$

Entre las limitaciones de esta investigación se encuentra el hecho de que la muestra se tomó de las mujeres trabajadoras que se acercaron voluntariamente a sus controles médicos en dos salas de un hospital, por lo que sus características fueron altamente variables entre el diagnóstico, el grado de cronicidad del dolor, el puesto de trabajo desempeñado, la jerarquía dentro de la organización, la antigüedad en el cargo, el nivel educativo, el estado civil y número de hijos, lo que no permitió que estas variables se consideraran para el análisis dentro de este estudio.

Se concluye que la baja satisfacción con la calidad de vida laboral favorece el catastrofismo y una alta satisfacción con la calidad de vida laboral facilita la aceptación del dolor crónico osteomuscular en las mujeres trabajadoras, por lo que mejorar las condiciones laborales y ofrecer un entorno saludable puede ser un factor determinante en su manejo y control. 


\section{REFERENCIAS}

1. Flores $N$, Jenaro $C$, González F, Garcia P. Análisis de la calidad de vida laboral en trabajadores con discapacidad. Ekaina-Zerbitzuan. 2010;(47):95-107.

2. Barragán $A$, Mejia $S$, Gutiérrez M. Dolor en adultos mayores de 50 años: prevalencia y factores asociados. Salud pública Méx. 2007;49(4):488-494.

3. Arango E, Buitrago L, Maya C, Portillo S, Uribe J, Vásquez E. Sindrome del túnel del carpo: aspectos clínicos y su relación con los factores ocupacionales. Rev CES Salud Pública. 2012;3(2):210-218.

4. Bedoya J, Vergara J, Araujo M, Ramos, E. Sindrome del túnel carpiano en la clínica universitaria San Juan de Dios de Cartagena, Colombia. Perfil epidemiológico, clínico y evaluación terapéutica. Rev cienc biomed. 2012;3(1): 49-57.

5. Sharifi-Mollayousefl A, Yazdchi-Marandi M, Ayramlou H, Heidari P, Salavati A, Zarrintan S. Assessment of body mass index and hand anthropometric measurements as independent risk factors for carpal tunnel syndrome. Folia Morphol. 2008;67(1):36-42.

6. Vargas PA, Orjuela ME, Vargas C. Lesiones osteomusculares de miembros superiores y región lumbar: Caracterización demográfica y ocupacional 2001-2009. Enferm Glob. 2013;12(4):2-12.

7. Covarrubias, A., Guevara U, Gutiérrez C, Betancourt J, Córdoba J. Epidemiologia del dolor crónico en México. Rev Mex Anest. 2010;33(4):207-213.

8. Aliaga L, Nebreda C, Ojeda A, Molà O, Galván J. Estudio observacional para conocer el perfil de los pacientes con dolor crónico musculoesquelético en tratamiento con la combinación de Tramadol $37,5 \mathrm{mg} /$ paracetamol $325 \mathrm{mg} 0$ Paracetamol $1 \mathrm{~g}$ o Metamizol $575 \mathrm{mg}$ (estudio PROFILE). Rev Soc Esp Dolor. 2013;20(3):122-131.

9. Kaufman D. Neurología clínica para psiquiatras. Amsterdam: Elsevier Masson; 2008.
10. González R, Hidalgo G, Salazar J, Preciado M. Elaboración y validación del instrumento para medir calidad de vida en el trabajo "CVT-GOHISALO". Cienc Trab. 2010;12(36):332-340.

11. Rodero B, Garcia-Campayo J, Casanueva B, López-Del-Hoyo Y, SerranoBlanco A, Luciano J. Veaselaircdhation of the Spanish version of the Chronic Pain Acceptance Questionnaire (CPAO) for the assessment of acceptance in fibromyalgia. Health qual life out. 2010;12(8):37-47.

12. Lasso C, Cruz A, Sánchez D, Toncel C. Dolor crónico en trabajadores de una empresa productora de comestibles. Cienc Salud. 2012;1(1):45-49.

13. Bosi de Souza T, Soares de Lima A, Prochnow A, Da Silva M, Petri J, De Souza J. Intensidad del dolor músculo-esquelético y la (in)capacidad para el trabajo en la enfermería. Rev Latino-Am Enfermagem. 2012;20(6):1-9.

14. Rodríguez A. Aspectos epidemiológicos de las patologías más frecuentes asociadas al sexo femenino. Informe Médico. 2009;11(6):331-334.

15. Valencillo $M$, Quevedo $A$, Lubo A, Dos Santos $A$, Montiel $M$, Camejo $M$, Sánchez $M$. Sintomas músculo-esqueléticos y estrés laboral en el personal de enfermería de un hospital militar. Salud trab. 2009;17(2):85-95.

16. Fuentes R, Lara E. Acoso laboral o mobbing y fibromialgia. Med Soc. 2011; $6(4): 29$.

17. Garcia-Campayo J, Rodero B. La catastrofización ante el dolor en la fibromialgia. Jano. 2011;(1772): 59-61.

18. Sánchez-Castillo C. Satisfacción de mujeres trabajadoras con la relación familia y trabajo. Rev Méd Inst Mex Seguro Soc. 2012;50(2):135-140.

19. Navarro A. ¿Mujeres proveedoras y jefas de familia?... Nuevas realidades rurales en localidades de la región zamorana. La Ventana. 2010;31(5):139171. 